\title{
Healthcare decision-making: past present and future, in light of a diagnosis of dementia
}

Karen Harrison Dening ${ }^{1 *} \mathrm{RMN}, \mathrm{RNLD}, \mathrm{RGN}, \mathrm{MA}, \mathrm{PhD}$, Head of Research \& Evaluation

Michael King ${ }^{3}$ Professor of Primary Care Psychiatry,

Louise Jones ${ }^{2}$ MB FRCP, Clinical Senior Lecturer

Elizabeth L Sampson ${ }^{2}$ MD MRCPsych, Reader

${ }^{1}$ Dementia UK, Second floor, Resource for London, 356 Holloway Road, London, N7 6PA

${ }^{2}$ Marie Curie Palliative Care Research Department, UCL Division of Psychiatry, 6th Floor, Maple House, 149 Tottenham Court Road, London W1T 7NF.

${ }^{3}$ Division of Psychiatry, Faculty of Brain Sciences, UCL

*Corresponding author

Tel: 02076974183

Karen.harrison-dening@dementiauk.org

Word Count: 5497 (including references, tables etc)

MeSH key terms: Dementia, advance care planning, decision-making, carers, end of life 


\begin{abstract}
End-of-life care policy in the UK states that all people should identify their needs, priorities and preferences for end-of-life care in the form of advance care planning. Advance care planning in dementia is less well developed than in some other disease groups such as cancer and arguably, may be more complex. A person with dementia may lose the capacity to make decisions associated with advance care planning early on in the course of the disease, requiring more involvement of family carers. This study explores the 'lived' context to health care decision-making of dyads (person with dementia and their carer) in respect of past, present and future healthcare decision-making.
\end{abstract}




\section{Background}

\section{Dementia}

Dementia is a progressive, irreversible neurodegenerative condition (Neale et al, 2001; Wilcock et al, 2008; Xie et al, 2008), and once diagnosed people will die with dementia regardless of the primary cause of death (Wilcock et al, 2008). Whilst many people with dementia (PWD) die of a medical complication, such as pneumonia or another infection, dementia itself can be the cause death; for example, general wasting, malnutrition, and dehydration when a person with dementia can no longer eat safely and move independently. However, the lack of recognition that dementia is a life limiting illness have led to neglect in addressing the end of life challenges for PWD and their carers (Sampson et al, 2006).

Raising public awareness of dementia and increasing diagnosis rates is a key focus of UK strategy and policy (DH, 2009; 2012). However, families affected by dementia can have many anxieties about what the future might hold both for the PWD as well as regarding their own future health and ability to care. Raising awareness about dementia is a double-edged sword; whilst increasing awareness, it may also reveal concerns about the possibility of developing dementia. In a recent poll, those over the age of 55 feared dementia (58\%) more than any other condition, including cancer (47\%) (Alzheimer Research UK, 2011). At the same time as this drive within dementia strategy and policy, the End-of-life care Strategy (DH, 2008) was launched stating all people should engage in advance care planning (ACP), identifying their priorities and preferences for end-of-life care, and that these should be respected and acted upon wherever possible.

\section{Advance care planning}

Preferences for future care are assumed to be based upon the principle of autonomy, whereby a person expects to retain personal control in making such decisions in advance of a time when they may experience ill health. ACP is a "process of discussion that usually takes place in anticipation of a future 
deterioration of a person's condition, between that person and a care worker" usually from a health care background (Henry \& Seymour, 2007). Implemented largely in the United States, Australia and Canada it involves a process of communication, facilitating a person's involvement in decisions about their current and future care, and putting in place plans to ensure that these preferences can be met when a person no longer has the capacity to express their wishes (Teno, 2010).

ACP is less well developed in Europe and the UK, where historically the legal status of advance statements have been less clear (van der Heide et al., 2003; Seymour et al., 2004). In England and Wales there is now recognition that under common law, a specific anticipatory statement (usually the advance refusal of medical treatment) has legal status. The Mental Capacity Act 2005 (Department of Constitutional Affairs, 2005) seeks to ensure that even those without mental capacity are enabled to make known their decisions, wishes and preferences for care and support, in anticipation that these will be carried out.

Professionals often rely on family carers to support decision-making for a person with dementia. However, little is understood about the families' beliefs and historic approaches to decision-making before the diagnosis of dementia was made. Whilst there is an increasing body of knowledge on proxy decision-making, there is little qualitative evidence of the 'lived' context to health care decision-making.

The aim of this study was to understand the 'lived' context to healthcare decision-making in the past, present and future of both the person with dementia and their family carer. This qualitative study was part of a larger study (Harrison Dening et al, in press) that examined whether family carers could predict the wishes and preferences for end-of-life care of the person with dementia for whom they cared.

\section{Method}

A qualitative approach was taken, drawing upon a naturalistic interpretive approach (Ritchie and Lewis, 2012; Topping, 2010). Content analysis was used as a qualitative research method for sorting, synthesising and analysing data from the interviews (Ritchie and Lewis, 2012). Content analysis is a 
useful approach to examining data within a given context and in the triangulation of data collected through mixed research methods (Given, 2008). Robson (2002) argues that this approach allows both the examinations of content and context to enable any research to link the qualitative data to quantitative 'outside variables' for example, gender.

\section{Participants}

A purposive sample (Polit \& Beck, 2009) of six dyads were selected from a larger cohort (n=60) study (Harrison Dening et al, in review) where we examined the ability of carers to predict future treatment preferences of the person with dementia using the Life Support Preferences Questionnaire (LSPQ). Six dyads: two that demonstrated a high level, two a medium level and two low level of agreement in the original study were purposively sampled for this qualitative study.

\section{Study location}

For pragmatic purposes, dyads were selected from a memory clinic service (BEH Mental Health Trust).

\section{Inclusion and exclusion criteria}

Person with dementia

We included PWD with Mini Mental State Examination (MMSE) (Folstein, et al, 1975) score of >20 out of 30 and the mental capacity to consent to and participate in the interview.

\section{Carer}

Carers were included if identified as next of kin or key decision maker for the person with dementia PWD.

Dyads were excluded if either the PWD or carer did not consent to take part and if either was unable to communicate sufficiently in English, as no funding was available for use of interpreters. 


\section{Recruitment}

Each potential dyad was contacted and a verbal explanation of the study was given by the researcher and followed up with a brief information sheet. A second contact was made after a seven day period to seek consent to inclusion in the study. All participants gave their signed consent.

\section{Data Collection}

Demographic details of age, gender, ethnicity, previous education, living situation and dyad relationship of all participants was recorded.

A brief, semi-structured interview schedule with three opened ended questions was designed to allow participants to answer questions with as much explanation as they chose followed by any prompts by the researcher or any further enquiry of each response as appropriate (see box 1). Interviews were digitally audio recorded to ensure that all aspects of discussions and conversation nuances were captured. In addition, field notes were taken to record observations of nonverbal communication such as worried expressions, raised eyebrows, etc. Participants were offered a choice of location for the interview, but all chose to be interviewed at home. All interviews were conducted between December 2013 and January 2014 by KHD and each lasted between 25 and 40 minutes.

\section{Box 1 approximately here}

\section{Data Analysis}

Interviews were transcribed verbatim, anonymised and encrypted. A content thematic approach was adopted incorporating a number of stages to systematically organise, reduce, refine and analyse the data (Braun and Clarke, 2006; Ritchie and Lewis, 2012). Emerging patterns were identified, manually coded and categorised from the data independently by KHD and ELS to ensure reliability and validity (Coffey 
\& Atkinson, 1996). The data tree and memos were then agreed upon by KHD and ELS.A sample of dyad interviews were independently blind coded and themed by a member of the Marie Curie Palliative Care Research Department.

\section{Results}

\section{Participant characteristics}

Six dyads agreed to participate, with one additional carer, the adult son of one dyad (Dyad D), included in the interview. The average age of the six participants with dementia was 77.6 years (ages raging from 70 to 88 years). The mean MMSE score was 24.8/30 (range 22-28). The average age of the seven carers was 73.4 years (range 49 to 85 ) (see table 1). All carers were spouses of the person with dementia, except for the additional adult son in dyad D (see table 2).

Table 1 approximately here

Table 2 approximately here

\section{Historic patterns of healthcare decision-making}

The first question explored how dyads had made healthcare decisions historically. Most felt they had not had to make any major healthcare decisions, until the diagnosis of dementia; such decision-making largely remained untested.

...there were very few major decisions we ever had to make.

(Carer, Dyad C, High agreement)

However, many proposed their approach would have been one of shared decision-making and spoke of guiding principles that would prevail. 
Well, we discuss it for a start and see what each one would say and then decide to come to a decision...I would never do anything without asking [name] about it or getting his opinion and he would do the same. Yes?

(PWD, Dyad C, High agreement)

...I don't think we would do anything the other didn't want to do...

(Carer, Dyad A, Medium agreement)

However, evidence revealed that many were autonomous in decision-making. In one instance each partner had attempted to exert health promoting behaviours on each other but the pressure to conform to healthier habits was resisted:

...he'd try to talk to me about it [stopping smoking]...it's got to come from me...

(Carer, Dyad B, High agreement)

Often there was one partner more dominant in decision-making though this was not always acknowledged within the dyad. One interview was joined by an adult child and a constant theme running through this interview was the spousal carer's dominance:

...well, she is very bossy [regarding decisions]...

(PWD, Dyad D, Low agreement)

\section{The effect of a diagnosis of dementia upon healthcare decision-making}

The second question explored the impact of the diagnosis of dementia on decision-making processes.

Carers talked of how they tried to influence the PWD to seek help. However, one carer felt there was little point as she felt nothing could be done afterwards even when the diagnosis was confirmed, the 
carer decided they would tell no one for fear of stigma. She felt this decision was shared though other family members saw this differently.

...we decided we were not going to mention it to anyone outside...only our son, possibly his wife...

(Carer, Dyad D, Low agreement)

Many spoke of dementia being the first time shared decision-making was tested, feeling the diagnosis marked a transition with historical decision-making roles being altered. One PWD had been the main decision-maker but because of dementia he now deferred this to his wife and a carer sought the support of other family members:

...when it is a difficult decision I ask my girls...[daughters]

(Carer, Dyad E, Low agreement)

Some PWD reflected on how stepping down from decisional responsibilities was a natural evolution rather than a conscious process:

I think gradually...I have, um....opted out of major decision-making...I'm not taking as much responsibility for our lives, as I would have in the past...it seems to be the natural thing...as though it's evolved...

(PWD, Dyad B, High agreement)

For most, an incident occurred to test the new order of decision-making. Carers wanted to 'soften' the the decision-making transition. It was the decision not to do something that they worried would 
challenge or upset the PWD. For example, not to go on holiday again as the change of environment created more difficulties than benefits.

...I have to decide...ohhh...well...holidays are a thing of the past now...I say to him how difficult it is to travel ... you know?

(Carer, Dyad E, Low agreement)

Carers felt that the decisions to be made often involved a compromise or loss to their own social lives, for example, no longer having holidays.

...I made that decision... [does not even mention the word to avoid distress] ...to avoid that situation because he gets upset... you know...

(Carer, Dyad E, Low agreement) Becoming the main decision-maker for some carers was felt wearisome. Whilst some felt compassion and self-sacrifice in making 'protective' decisions to 'soften the reality' for the person with dementia, it often came at a cost.

It is probably the practical...everyday decisions...day to day decisions that I have to make...it is very wearing for me...it is very stressful for me...

(Carer, Dyad F, Medium agreement) [the burden of decision-making]...to me...most of it... all of it really...

(Carer, Dyad E, Low agreement)

Carer's felt a drive to 'protect' the PWD due to a fear of what the future might hold should something happen to them, what would happen to the PWD? 
...if he can't decide now...it will be worse in the future, won't it?... while I am carer...then I make the decisions but if I come to a position where he [PWD] is now and I can't make decisions...

(Carer, Dyad E, Low agreement)

\section{Future healthcare decision-making in light of the dementia diagnosis}

The final question sought to explore what healthcare and/or treatment decisions participants may need to make in the future.

There were certain decisions carers felt may be required at some point but were unsure when or how these might arise. The issue of where care was to be delivered if they became unwell or unable to care arose in several interviews. Carers felt that admission to a care home may be a decision that would present itself in a crisis rather than something to plan for:

If that happened [carer ill health] ...it would change the entire set up. The only option would be for [name PWD] to go into a home...

(Carer, Dyad C, High agreement)

I really don't know... about nursing homes... but if it has to come to that, and there is no other option...to decide... but at the moment I just block it out of my head, I don 't want to think about it because...it's hard...

(Carer, Dyad E, Low agreement)

This, perhaps inevitably, resulted in a strong reaction from the person with dementia who, whilst recognising their failing powers, could not conceive of a time when this decision would ever be appropriate or desired.

[dementia] ...it's not going to get worse...here I am, here I am going to stay... 
(PWD, Dyad E, Low agreement)

There were divided views on planning ahead and if so, how to do it. Some had limited knowledge of the legal system to support decision-making when capacity was lost, including ACP, Lasting Powers of Attorney.

Mum doesn't want to do it... [LPA]...

(Adult son, Dyad D, Low agreement)

[LPAs]...I didn't say not at all...I wouldn't do it until much later on I think...I see it as something you hand over when its necessary, not before...

(Carer, Dyad D, Low agreement)

However, some were considering making plans for the future and indicated where they were in this:

...we've thought about it, I've got all the paperwork...but I have got to talk to the kids before I can do it...

(Carer, Dyad A, Medium agreement)

Even if they were aware of the legal processes many had limited knowledge about the course of dementia and its prognosis and what effect this could have on decision-making. Some carers felt that it was hard work to support and influence decision-making for the PWD:

...there are certain milestones...that people [with dementia] are going to go through...I am not sure we fully understand what we need to do... more difficult [in dementia] to plan and understand the progression...you don't really know what is going on...

(Adult son, Dyad D, Low agreement) 
Decision-making and living with dementia was often difficult and meant only a 'day-to-day' or 'day-byday' approach was possible with the bigger decisions seen as too far ahead to be able to emotionally consider or practically tackle:

...day-to-day decisions that I have to make...it is very wearing...

(Carer, Dyad F, Medium agreement)

I can't make decisions...well, I can make decisions...but erm, really, I have to take each day as it comes...

(Carer, Dyad E, Low agreement)

When speaking of trust in professional decision-making at end of life several participants spoke of the concerns surrounding the Liverpool Care Pathway (LCP).

...there are several things wrong with the Liverpool...you know...that you are getting nurses making decisions, junior doctors making decisions without full consultation with relations and the senior...physicians...

(Carer, Dyad D, Low agreement) ...in the light of all the... misguidance... on balance I was still against the Liverpool Care Pathway unless there was much more control...

(PWD, Dyad D, Low agreement)

The carer had the final say on the LCP issue by stating a concern that external factors may override the wishes of the dying person.

Also there may be other considerations in the professional's mind like number of beds, or meeting targets, erm...those things are around though unspoken...so... 
(Carer, Dyad D, Low agreement)

Experience of a death of a close family member often influenced participants' understanding of issues around end-of-life care, though interestingly, none had used this experience to consider their own situations.

[talking of a sister] ...she didn't want to talk about it so we didn 't..it is such a distressing thing...she was in a clinic...so I didn't feel I had to make any decisions...

(Carer, Dyad D, Low agreement)

\section{Discussion}

\section{Summary of key findings}

1. Although dyads had a history of making decisions together this had not usually involved healthcare decisions.

2. Dyads describe how decision-making changes after a diagnosis of dementia.

3. Carers recognise increasing responsibility for decision-making after the diagnosis of dementia.

4. The 'day to day' decision-making becomes burdensome so much so that it distracts carers from focusing their attention to long term planning.

5. Even when families had previous experience of supporting other family members at end-of-life this did not seem to stimulate them to establish their own Lasting Power of Attorney's and Advance Care Plan's.

Participants were aware of change to decision-making soon after the diagnosis of dementia. As in other studies (Smebye et al., 2012), some PWD recognised these changes and letting others take over decision-making was seen as acceptable a natural process. However, others saw no need to change the 
current status and that the future would be no different from the here and now. However, this may be indicative of PWD having difficulty imagining their future self (Harrison Dening et al. 2012).

Carers recognised increasingly being faced with having to assume responsibility for the decisions and welfare of the PWD. Such decision-making came with an 'emotional price tag', finding that day-to-day decision-making all consuming with little energy for medium to long term decisions and planning. Feinberg and Whitlach (2002) also identified carers were most concerned about 'getting through each day' and this influenced how much weight they gave to considering issues lying ahead in the future. Sampson \& Clark (2015) studied male carers' decision-making and found that negotiating the complexity of everyday decisions detracted from considering longer term issues generating greater uncertainty and anxiety. Burdensome decision-making is not just related to getting through those that present day to day but also how the decisions made often requires carers to put their own wishes secondary to those of the PWD, which adds to perceptions of burden (Quinn et al. 2015). Carers made every effort to ensure the PWD was 'protected' through undertaking the day-to-day decisions to preserve the dignity of the PWD but in doing so often neglected their own needs.

Participants had little experience of major healthcare decision-making until the diagnosis of dementia, so as such this had remained largely untested. Dyads held the belief that when the need to make decisions arose that, shared, family decision-making would prevail. The diagnosis of dementia had a marked impact on decision-making processes; there was often a shift to the carer being required to make many more decisions on behalf of the PWD than would have historically been the case. Such a shift of decisional responsibility to the historically less dominant partner added further burden in their new caring role with the mundane day-to-day ones being unrelenting and burdensome.

Participants spoke of the trust they had in others to make decisions, feeling that they knew them well enough to be of good support if decisions were required. It is argued that knowing the person with 
dementia will enable a carer (and others) to understand their values and individual decision-making patterns (Clarke et al., 2003; Bruce and Schweitzer, 2008). Indeed, most of dementia care is rooted in the principle of knowing the person with dementia as being the basis for good person-centred care (Kitwood, 1997). Whilst the argument for autonomy in decision-making wherever possible is made for PWD, in reality decisions are often made within a family or relationship context (O'Connor and Purves, 2009). Smebye et al. (2012) found that various family bonds, positive or negative, influenced decisionmaking and affected changes in roles and power dynamics as a result of the dementia. Many also spoke of their trust in doctors, expect them not only to guide them but also decide for them as they were the expert. Other research found that carers and PWD viewed doctors as being supportive of their preferences (Noh \& Kwak, 2016). It could be that many people of this generation are more willing deferring responsibility for decision-making, especially to doctors.

Finally, uncertainty prevailed when considering decisions they may need to make the future. To be able to plan ahead requires appropriate information to weigh up the pros and cons of various options. Thus carers and PWD need information on the possible life course of dementia and its impact upon the individual and the wider family. Many dyads felt they lacked information with which to consider their future, although studies have shown that such information given in managed chunks is supportive of carer decision-making (Wald et al., 2003; Robinson et al., 2013; Livingston et al., 2010).

Some participants had experience of life-limiting conditions in other family members, including both providing care and being appointed as decision makers within LPAs. However, this had not influenced them to make LPAs or ACPs for themselves. This contradicts findings of an observational cohort study of older people who experienced end-of-life care of others demonstrated greater readiness to participate in ACP (Amjad et al, 2014). Carers are often expected to act as "proxies" for PWD and make difficult and emotionally demanding choices and decisions, for example, regarding resuscitation (Hirschman et al., 2006) or care home placement (Davies \& Nolan, 2004; Ryan \& Scullion, 2000). Carers of PWD 
perceive high levels of carer related burden, distress and depression (Chan et al., 2013) and that a lack of information on the disease trajectory and its prognosis leaving them unprepared to make decisions on end-of-life care on behalf of their relative (Lord et al., 2015).

\section{Strengths and limitations of this study}

The study sample was small and may not be representative of this population. However, through purposive recruitment from a larger cohort we were able to represent families with a range of levels of concurrence in future decision-making; this enabled us to capture a wide range of views. It is the first study of its kind that aimed to seek an understanding of the 'lived' context to historic, current day and future healthcare decision-making of dyads in the advent of a diagnosis of dementia.

\section{Clinical implications}

Families require ongoing support and guidance on decision-making following a diagnosis of dementia. Signposting and the provision of information may not be sufficient to enable families to understand the changes dementia may have on their usual strategies for decision-making and to prepare for future eventualities.

There is often a belief that carers and PWD speak with 'one voice' but this cannot be assumed. This is also supported by our previous work (Harrison Dening, et al. 2012; Harrison Dening, et al. in press). Families affected by dementia are likely to require ongoing support to develop plans and adapt to changes in decision-making patterns as the illness progresses.

\section{Conclusion}

All families affected by dementia should have access to post diagnostic support and counselling that takes into account changes that occur in decision-making patterns within their relationships. Clinicians, when considering how they may support families in building their resilience in living with dementia, 
need to understand previous relationship strengths and weaknesses and historic family decision-making processes as this may either indicate qualities on which to maximise or may highlight areas for increased support.

In supporting ACP for the person with dementia clinicians will need to explore the couple's approach to and ability to make decisions (Boyle, 2013); this should consider any carer tendencies to dominate or assume that they know best. Carers require support for day-to-day decision-making that maximises the strengths of the PWD for as long as possible. This will need to take account of a PWD's wish to retain a sense of control and dignity while at the same time balancing carers' needs as the relationship changes because of dementia. 


\section{References}

Alzheimer's research UK (2011) www.alzheimersresearchuk.org/alzheimers-research-uk-launch/

[Accessed 08 February 2016].

Amjad, H., Towle, V. \& Fried, T. (2014) Association of Experience with Illness and End-of-Life Care with Advance Care Plannning in Older Adults. JAGS, 62: 1304-1309.

Boyle, D. (2013) 'She's usually quicker than the calculator': financial management and decision-making in couples living with dementia. Health and Social Care Community, 21 (5): 554-562.

Braun, V. \& Clarke, V. (2006) Using thematic analysis in psychology. Qualitative Research in Psychology 3 (2): 77-101.

Brooker, D. (2007) Person-Centred Dementia Care: Making Services Better (Bradford Dementia Group Good Practice Guides), London: Jessica Kingsley Press.

Bruce, E. \& Schweitzer, P. (2008) Working with life-history. In: Downs, M. \& Bowers, B. (Editors) Excellence in dementia care, New York: Open University Press.

Bryman, A. \& Burgess, R. (Editors) (1994) Analyzing Qualitative Data, London: Routledge.

Chan, D., Livingston, G., Jones, L. \&, Sampson, E.L. (2013) Grief reactions in dementia: a systematic review. International Journal of Geriatric Psychiatry, 28(1): 1849-58.

Clarke, A., Hanson, E.J. \& Ross, H. (2003) Seeing the person behind the patient: enhancing the care of older people using a biographical approach. Journal of Clinical Nursing, 12: 697-705.

Coffey, A. \& Atkinson, P. (1996) Making sense of qualitative data. Thousand Oaks, CA: Sage.

Davies, S. \& Nolan, M. (2004) 'Making the move': relatives' experiences of the transition to a care home. Health \& Social Care in the Community,12(6):517-26.

DH (2005) Creating a Patient Led NHS: Delivering the NHS Improvement Plan, London: Department of Health.

DH (2008) End-of-Life Care Strategy, London: Department of Health.

DH(2009) Living Well with Dementia: A National Dementia Strategy, London: Department of Health.

DH (2012) Prime Ministers Challenge on Dementia: Delivering major improvements in dementia care and research by 2015, London: Department of Health.

Department of Consitutional Affairs. Mental Capacity Act 2005.

Feinburg, L.F. \& Whitlach, C.J. (2002) Decision-making for persons with cognitive impairment and their family caregivers. American Journal of Alzheimer's Disease and Other Dementias, 17 (4): 237 244. 
Folstein, M.F., Folstein, S.E., \& McHugh, P.R. (1975) 'Mini-Mental state'. A practical method for grading the cognitive state of patients for the clinician. Journal of Psychiatric research, 12: 189-198.

Given, L.M. (Editor) (2008) The SAGE Encyclopaedia of Qualitative Research Methods. London: SAGE Publications.

Harrison Dening, K., Jones, L. \& Sampson, E.L. (2012) Preferences for end-of-life care: A nominal group study of people with dementia and their family carers. Palliative Medicine, DOI:

$10.1177 / 0269216312464094$

Harrison Dening, K, King, M., Jones L.,Vickerstaff, V. \& Sampson E.L. (in press) Advance care planning in dementia: Do family carers know the treatment preferences of people with dementia? PlosOne.

van der Heide, A., Deliens, L., Faisst, K., Nilstun, T., Norup, M., Paci, E, et al. (2003) End-of-ife decision-making in six European countries: descriptive study. Lancet, 362:345-50.

Henry, C. \& Seymour, J. (2007) Advance Care Planning: A guide for health and social care staff. London: National End of Life Care Programme,Department Of Health.

Kitwood, T. (1997) Dementia Reconsidered: The person comes first, Buckingham: Open University Press.

Livingston, G., Leavey, G., Manela, M., Livingston, D., Rait G., Sampson, E.L., Bavishi, S., Shahriyarmolki, K. \& Cooper, C. (2010) Making decisions for people with dementia who lack capacity: qualitative study of family carers in UK. BMJ, 341: c4184. doi: 10.1136/bmj.c4184.

Lord, K., Livingston, G., \& Cooper, C. (2015) A systematic revioew of barriers and facilitators to and interventions for proxy decision-making by family carers of people with dementia. International Psychogeriatrics, 27(8): 1301-12.

Mason, J. (2002) Qualitative Researching, $2^{\text {nd }}$ edition, London: Sage Publications.

Miles, M.B. \& Huberman, A.M. (1994) Qualitative Data Analysis: an Expanded Sourcebook, Thousand Oaks, California: Sage Publications.

Noh, H. \& Kwak, J. (2016) End-of-life decision-making for persons with dementia: Proxies' perceptions of support. Dementia, pii: 1471301216643473 [Epub ahead of print].

O’Connor, D. \& Purves, B. (2009) Decision-making, personhood and dementia, London: Jessica Kingsley Publishers.

Polit, D.F. \& Beck, C. (2009) Essentials of Nursing Research: Appraising Evidence for Nursing Practice. $7^{\text {th }}$ edition, Philadelphia: Lippincott William \& Wilkins.

Quinn, C., Clare, L. \& Woods, R.T. (2015) Balancing needs: the role of motivations, meanings and relationship dynamics in the experience of informal caregivers of people with dementia. Dementia, 14(2): 220-237.

Ritchie, J. \& Lewis, J. (editors) (2012) Qualitative Research Practice: A Guide for Social Science Students and Researchers. London: Sage Publications. 
Robinson, L., Dickinson, C., Bamford, C., Hughes, J. \& Exley, C. (2013) A qualitative study: professionals' experiences of advance care planning in dementia and palliative care, 'a good death in theory but...'. Palliative Medicine, 27 (5): 401-408.

Robson, C. (2002) Real World Research, $2^{\text {nd }}$ edition, Oxford: Blackwell.

Ryan, A.A, \& Scullion, H.F. (2000) Nursing home placement: an exploration of the experiences of family carers. Journal of Advanced Nursing, 32(5):1187-95.

Saks, M. \& Allsop, J. (2007) Researching Health: Qualitative, Quantitative and Mixed Methods, London: Sage Publications.

Sampson, E.L., Gould, V., Lee, D. \& Blanchard, M. (2006) Differences in care received by patients with and without dementia who died during acute hospital admission: a retrospective case note study. Age and Ageing, 35 (2): 187-189.

Sampson, M.S. \& Clark, A. (2015) 'Deferred or chickened out?' Decision-making among male carers of people with dementia. Dementia. DOI: 1471301214566663.

Smebye, K.L., Kirkevald, M. \& Engedal, K. (2012) How do persons with dementia participate in decisionmaking related to health and daily care? A multi-sae study. BMC Health Services Research, 12: 241, doi: $10.1186 / 1472-6963-12-241$.

Teno, J.M. (2010) Advance directives for nursing home residents: achieving compassionate, competent, cost-effective care. The Journal of the American Medical Association, 283(11):1481-2.

Topping, A. (2010) The Quantitative-Qualitative Continuum, in GERRISH, K., \& LACEY, A. (editors) The Research Process in Nursing, $6^{\text {th }}$ edition, Oxford: Wiley-Blackwell Publishing.

Wald, C., Fahy, M., Livingston, G. \& Walker, Z. (2003) What to tell dementia caregivers- the rule of threes/ International Journal of Geriatric Psychiatry, 18: 313-317.

Wilcock, J., Froggatt, K. \& Goodman, C. (2008) End of Life Care. In: DOWNS M. \& BOWERS, S. (editors) Excellence in Dementia Care: Research into Practice, Maidenhead: Open University Press.

Xie, J., Brayne, C. \& Matthews, F.E. (2008) Survival times in people with dementia: analysis from population based cohort study with 14 year follow-up. BMJ, 336 (7638): 258-262. 


\section{Box 1: Brief Interview Guide}

1. How have you made decisions about healthcare and/or treatment wishes in the past?

2. What changes to this decision-making process (if any) do you see the diagnosis of dementia has made?

3. What healthcare and/or treatment decisions may you need to make in the future now that there is a diagnosis of dementia made (for you/your family member)?

\section{Table 1: $\quad$ Characteristics of dyads}

\begin{tabular}{|c|c|c|}
\hline & $(n=6)$ & Carers $(\mathrm{n}=7)^{*}$ \\
\hline Age (mean [range]) & $77.6[70-88]$ & $73.4[49-85]$ \\
\hline Gender (male) & 3 & 4 \\
\hline MMSE (mean [range]) & $24.8[22-28]$ & \\
\hline Diagnosis (ICD 10) & & \\
\hline F00.1 (Alzheimer's late onset) & 5 & - \\
\hline F00.2 (atypical or mixed type Alzheimer's) & 1 & - \\
\hline Ethnicity & & \\
\hline White British & 3 & 4 \\
\hline White other & 3 & 3 \\
\hline Previous education & & \\
\hline Left school $\leq 14$ years & 2 & 1 \\
\hline Left school $\geq 14$ years & 4 & 6 \\
\hline Living situation of PWD & & \\
\hline Spouse & 6 & - \\
\hline Relationship to PWD & & \\
\hline
\end{tabular}




\begin{tabular}{|l|l|l|}
\hline Spouse & - & 6 \\
Adult child & & \\
\hline
\end{tabular}

Note: $*=$ one interview involved an adult child in addition to the spouse. PWD $=$ Person With

Dementia. MMSE $=$ Mini Mental State Examination. ICD $10=$ International Disease Classification

Table 2: $\quad$ Participants level of agreement using modified LSPQ

\begin{tabular}{|l|l|l||}
\hline Dyad ID & LSPQ score & Level of agreement \\
\hline A & $6 / 9$ & Medium $(\mathrm{M})$ \\
\hline B & $9 / 9$ & High $(\mathrm{H})$ \\
\hline C & $8 / 9$ & High \\
\hline D & $2 / 9$ & Low $(\mathrm{L})$ \\
\hline E & $3 / 9$ & Low \\
\hline F & $6 / 9$ & Medium \\
\hline
\end{tabular}


